\title{
Shear viscosity of QGP and the anisotropic flows within an event by event transport approach
}

S. Plumari ${ }^{1,2}$, G. L. Guardo ${ }^{2}$, A. Puglisi ${ }^{1,2}$, F. Scardina $^{1,2}$ and V. GRECO ${ }^{1,2}$

${ }^{1}$ Department of Physics and Astronomy, University of Catania,

Via S. Sofia 64, I-95123 Catania, Italy

${ }^{2}$ INFN-Laboratori Nazionali del Sud, Via S. Sofia 62, I-95123 Catania, Italy

\begin{abstract}
We have employed a relativistic kinetic transport approach that incorporates initial state fluctuations to study the effect of a temperature dependent shear viscosity to entropy density ratio $\eta / s(T)$ on the build-up of the anisotropic flows $v_{n}\left(p_{T}\right)$. We find that at LHC energies and for ultra-central collisions $(0-0.2 \%)$ the $v_{n}\left(p_{T}\right)$ have a stronger sensitivity to the T dependence of $\eta / s$ in the QGP phase and this sensitivity increases with the order of the harmonic n. Moreover we have studied the correlation between the initial spatial anisotropies $\epsilon_{n}$ and the final flow coefficients $\left\langle v_{n}\right\rangle$ for different centralities and for the two beam energies. The study shows that at LHC energies there is more correlation than at RHIC energies. In particular at LHC energies and for ultra-central collisions the linear correlation coefficient $C\left(\epsilon_{n}, v_{n}\right) \approx 1$ for $n=2,3,4$ and 5 suggesting that the $\left\langle v_{n}\right\rangle$ are strongly related to the initial value of $\epsilon_{n}$.
\end{abstract}

\section{Introduction}

One of the most surprising results obtained in the experiments conducted at $\mathrm{RHIC}$ and more recently at the LHC is that the matter created in these 
heavy ion collisions behaves as an almost perfect fluid with a very low $\eta / s$ ratio. The observable indicating such a low value for $\eta / s$ is the so called elliptic flow, $v_{2}$. The elliptic flow is the second coefficient in the Fourier expansion of the azimuthal angle distribution of emitted particles and it a measure of the azimuthal asymmetry in momentum space. The determination of the $\eta / s$ ratio of the QGP is one of the main challenges in the relativistic heavy ion physics. The comparison between experimental data and theoretical calculations within viscous hydrodynamics and transport approach [1-4] have shown that this large value of $v_{2}$ is consistent with a very low $\eta / s$. The average value extracted is close to $1 /(4 \pi)$ which is the lower bound conjectured for strongly interacting systems, $\eta / s=1 / 4 \pi[5]$.

The possibility to measure event-by-event the angular distribution of emitted particle has made possible to extend this analysis to high order Fourier coefficients $v_{n}$ with $n>2$. A shown by theoretical calculations the sources for these momentum anisotropies $v_{n}$ are fluctuations in the initial geometry [6-9]. The comparison between experimental data and event-by-event viscous hydrodynamic calculations have shown that the expected range of viscosity explored from RHIC to LHC energies is about $4 \pi\langle\eta / s\rangle \sim 1-3[8,9]$. However there are several theoretical indications that $\eta / s$ should have a particular temperature dependence $[10,11,13-15]$. As an example in Fig.1 shows a collection of theoretical results about the $\mathrm{T}$ dependence of $\eta / s$. Therefore a phenomenological estimation of the T dependence of $\eta / s$ could give more information information if the matter created in these collisions undergoes a phase transition $[10,11,16]$. More recently within viscous hydrodynamic calculation have been studied the correlations between the initial anisotropies, measured by the eccentricities $\epsilon_{n}$, and the final anisotropies in momentum space $v_{n}$ [17-19]. In general it has been shown that the degree of correlation between $\epsilon_{n}$ and $v_{n}$ for a fixed centrality decrease with the order of the harmonic $n$.

In this proceeding we show the effects of the $\eta / s(T)$ on the $v_{n}\left(p_{T}\right)$ and we study the correlations between the initial $\epsilon_{n}$ and the final $v_{n}$ by using a cascade approach with initial state fluctuations [20].

\section{Kinetic approach at fixed $\eta / s(T)$}

We have employed a relativistic transport code developed in these years to perform studies of the dynamics of heavy-ion collisions at both RHIC and LHC energies [3, 20,25-28]. We solve numerically the RBT equation using the so called test particle method while for the collision integral we use the Monte Carlo methods, for details see $[3,25,29]$. 

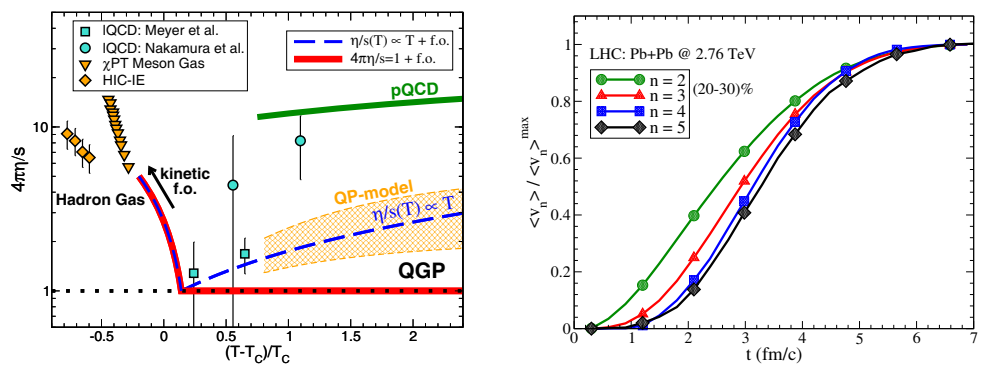

Figure 1: Left panel: $\eta / s(T)$ parametrizations. The light blue symbols are 1QCD data $[14,21]$. The orange symbols are for chiral perturbation theory $[12,13]$ and the phenomenological analysis for HIC at intermediate energy (HIC-IE) [22, 23]. The green line refers to pQCD calculations [24]. Right panel: $\left\langle v_{n}\right\rangle /\left\langle v_{n}\right\rangle^{\max }$ as a function of time for $(20-30) \%$. Different symbols are for different harmonics $n$.

In the standard use of the transport theory one fixes the microscopical details of the scattering like cross sections of the processes to study the effect on the observables. In the following discussion we invert the description not in terms of fixed cross section $\sigma_{t o t}$ but in terms of fixed $\eta / s$. We simulate a system at fixed $\eta / s(T)$ by evaluating in each grid cell the total cross section $\sigma_{\text {tot }}$ needed to have the wanted local viscosity. For a pQCD inspired cross section where $d \sigma / d t \sim \alpha_{s}^{2} /\left(t-m_{D}^{2}\right)^{2}$ with $m_{D}$ being the screening mass regulating the angular dependence of the cross section, the $\eta / s$ in the Chapmann-Enskog theory is given by the following expression:

$$
\eta / s=\frac{1}{15}\langle p\rangle \tau_{\eta}=\frac{1}{15} \frac{\langle p\rangle}{g(a) \sigma_{t o t} \rho}
$$

with $a=m_{D} / 2 T$ while $g(a)$ is a function that take into account for the pertinent relaxation time $\tau_{\eta}^{-1}=g(a) \sigma_{\text {tot }} \rho$ associated to the shear transport coefficient. The function $g(a)$ it is given by: $g(a)=$ $\frac{1}{50} \int d y y^{6}\left[\left(y^{2}+\frac{1}{3}\right) K_{3}(2 y)-y K_{2}(2 y)\right] h\left(\frac{a^{2}}{y^{2}}\right)$ where $K_{n}$-s are the Bessel functions. The function $h$ relate the transport cross section to the total cross section $\sigma_{t r}(s)=\sigma_{t o t} h\left(m_{D}^{2} / s\right)$ with $h(\zeta)=4 \zeta(1+\zeta)[(2 \zeta+1) \ln (1+1 / \zeta)-2]$.

In Fig. 1 it shown shown the $\mathrm{T}$ dependence expected for $\eta / s$. The solid and dashed lines are the two parametrization considered in this work. As shown the $\eta / s$ ratio is expected to increase at low temperature towards the estimated value for hadronic matter $4 \pi \eta / s \approx 6$. Within this approach we have a self-consistent implementation of the kinetic freeze-out with a smooth switching-off of the scattering rates at low temperature, see [3, 20,27]. 

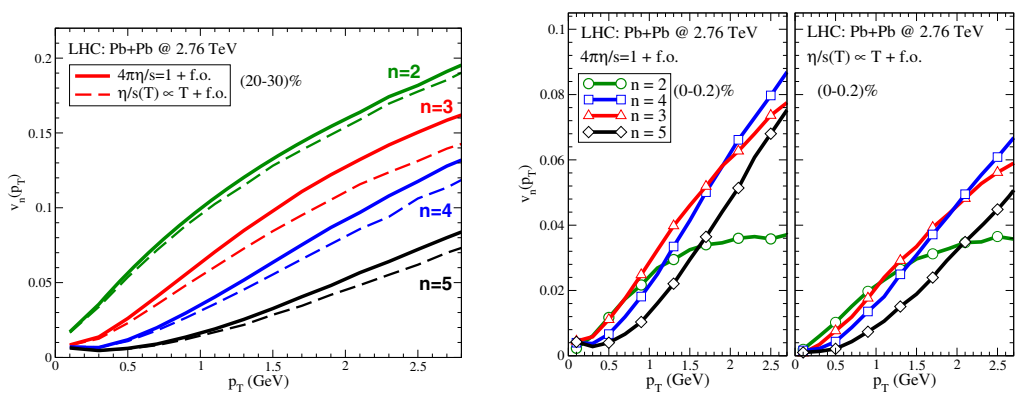

Figure 2: Left panel: $v_{n}\left(p_{T}\right)$ at mid rapidity for $(20-30) \%$. Solid lines correspond to $\eta / s=(4 \pi)^{-1}$ in QGP phase while dashed lines to $\eta / s \propto T$ in QGP phase. Right panel: comparison between $v_{n}\left(p_{T}\right)$ at mid rapidity for $(0-0.2) \%$ and for different $\mathrm{T}$ dependence of $\eta / s$. Different symbols are for different harmonics $n$.

\section{Anisotropic flows $v_{n}\left(p_{T}\right)$ and correlations}

We will show the results for $A u+A u$ collisions at $\sqrt{s_{N N}}=200 \mathrm{GeV}$ produced at RHIC and $\mathrm{Pb}+\mathrm{Pb}$ collisions at $\sqrt{s_{N N}}=2.76 \mathrm{TeV}$ at LHC. The implementation of initial state fluctuations in a transport cascade approach is made by using a Monte-Carlo Glauber model. Within this approach we determine initial distribution of the participant nucleons. The discrete distribution for the nucleons is converted in a smooth one by assuming for each nucleon a gaussian distribution. The width it has been fixed to $\sigma_{x y}=0.5 \mathrm{fm}$. The density in the transverse plane $\rho_{T}(x, y)$ is given by the following sum $\rho_{T}(x, y) \propto \sum_{i=1}^{N_{\text {part }}} \exp \left[-\frac{\left(x-x_{i}\right)^{2}+\left(y-y_{i}\right)^{2}}{2 \sigma_{x y}^{2}}\right]$. In this way we generate an initial density profile that change event-by-event. For the initialization in momentum space at RHIC (LHC) energies we have considered a thermalized spectrum for partons with transverse momentum $p_{T} \leq p_{0}=2 \mathrm{GeV}(3 \mathrm{GeV})$. The initial local temperature in the transverse plane $T(x, y)$ is given by standard thermodynamical relation $\rho_{T}(x, y)=\gamma T^{3} / \pi^{2}$ with $\gamma=40$. The central region of the fireball for mid peripheral collision can reach temperature $T \approx 300 \mathrm{MeV}$ at RHIC and $T \approx 400 \mathrm{MeV}$ at LHC. While for partons with $p_{T}>p_{0}$ we assume the spectrum of non-quenched minijets according to standard NLO-pQCD calculations [30]. The initial time of the simulation is $\tau_{0}=0.6 \mathrm{fm} / \mathrm{c}$ for RHIC and $\tau_{0}=0.3 \mathrm{fm} / \mathrm{c}$ for LHC. For a detailed discussion about the implementation of the initial condition see [20]. In the right panel of Fig.1 it is shown the time evolution of the $v_{n}$ normalized to their maximum. As shown different $v_{n}$ have a different formation time. In particular harmonics with larger $n$ develop later [20]. This imply that differ- 

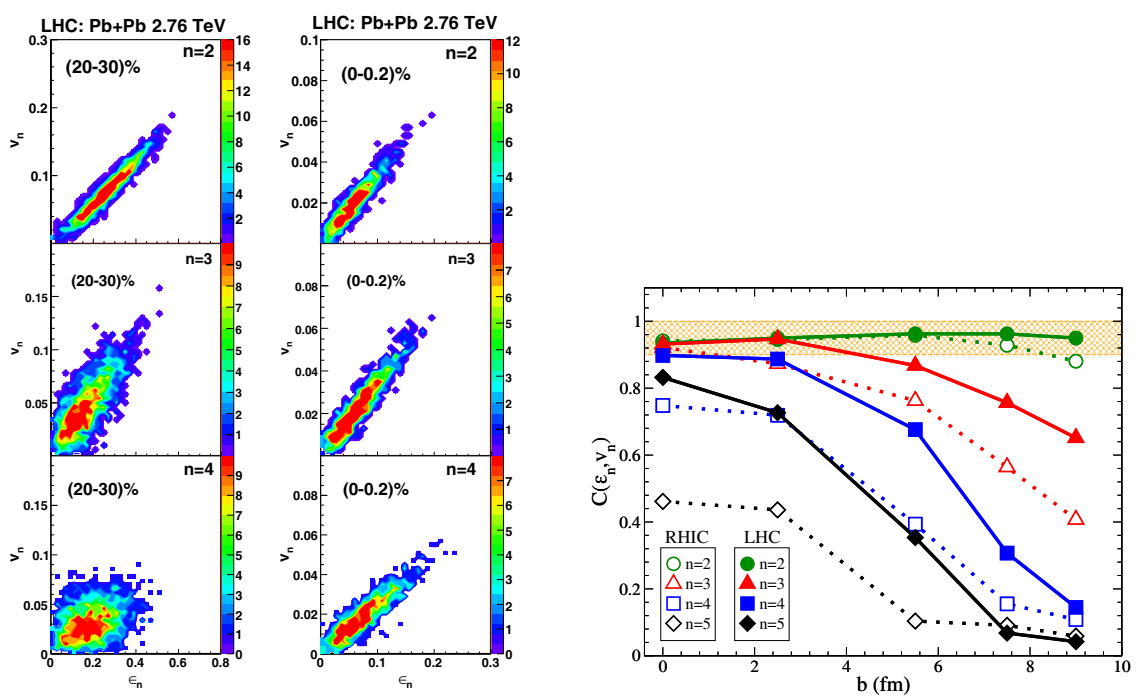

Figure 3: Left panel: Correlation plots between the initial eccentricities $\epsilon_{n}$ and final $v_{n}$ at LHC energies and for two centralities $(20-30) \%$ and $(0-0.2) \%$. In these calculations we have considered $\eta / s=1 /(4 \pi)$ for hight temperature and the kinetic f.o. at lower temperature (see red solid line in Fig.1). Right panel: $C\left(\epsilon_{n}, v_{n}\right)$ as a function of the impact parameter $b$. Different symbols refer to different harmonics $n$. The dotted (solid) lines are for RHIC (LHC).

ent $v_{n}$ develop in different stages of the fireball evolution and therefore they can probe different values of the $\eta / s$ of the QGP. This means that each $v_{n}$ have a different sensitivity to the $\mathrm{T}$ dependence of $\eta / s(T)$. As shown in the left panel of Fig.2 for mid-peripheral collisions the effect of the increase of $\eta / s$ at high temperature is to reduce the $v_{n}\left(p_{T}\right)$ and the reduction is about of $10 \%$. In ultra-central collisions (see right panel of Fig.2) the sensitivity to $\eta / s(T)$ is strongly enhanced and we observe a reduction of $v_{n}\left(p_{T}\right)$ of about $30-35 \%$ for $n>3$. This sensitivity increase with the order of the harmonics $n$. Moreover the $p_{T}$ dependence of $v_{n}\left(p_{T}\right)$ change with the centrality. In fact as shown in Fig. 2 for mid-peripheral collisions we observe that larger is $n$ smaller is the corresponding anisotropic flow. In ultra-central collisions the ordering occurs only at low $p_{T}<1 \mathrm{GeV}$ where $v_{n}\left(p_{T}\right) \propto p_{T}^{n}$. At higher $p_{T}$ the $v_{n}\left(p_{T}\right)$ are approximatively linear in $p_{T}$ for $n \geq 3$ while we observe a saturation for $v_{2}\left(p_{T}\right)$ similar to that observed experimentally.

In the following discussion we show the results of correlations between the initial $\epsilon_{n}$ and the final $v_{n}$. The following results have been obtained with $N_{\text {event }}=1000$ events for each centrality class with a total number of 
test particle per event of $N_{\text {test }}=2 \cdot 10^{6}$. In the correlation plots shown in left panel of Fig.3 we compare the degree of correlation between $\epsilon_{n}$ and $v_{n}$ for $(0-0.2) \%$ and $(20-30) \%$. The results are for LHC energies. In the upper panel we observe a strong linear correlation between $\epsilon_{2}$ and $v_{2}$ for both centralities. In agreement with viscous hydrodynamics [19]. In the middle and lower panel of Fig.3 it has been shown the correlation between $\epsilon_{3}$ and $v_{3}$ and between $\epsilon_{4}$ and $v_{4}$ respectively. For both cases we observe a reduction of the degree of correlation with the centrality. The correlation between $\epsilon_{3}$ and $v_{3}$ for all the centralities is weaker with respect to that obtained for $\epsilon_{2}$ and $v_{2}$. Furthermore the $v_{4}$ shows a even a weaker degree of correlation with the initial $\epsilon_{4}$ where for mid-peripheral collisions approximatively they are decorrelated. Similar behavior we observe at RHIC energies. To quantify the degree of correlation between $\epsilon_{n}$ and $v_{n}$ we have studied the linear correlation coefficient $C\left(\epsilon_{n}, v_{n}\right)$ as a function of the impact parameter for both RHIC (dotted lines) and LHC energies (solid lines). Where $C\left(\epsilon_{n}, v_{m}\right)$ is given by:

$$
C\left(\epsilon_{n}, v_{m}\right)=\frac{\sum_{i}\left(\epsilon_{n}^{i}-\left\langle\epsilon_{n}\right\rangle\right)\left(v_{m}^{i}-\left\langle v_{m}\right\rangle\right)}{\sqrt{\sum_{i}\left(\epsilon_{n}^{i}-\left\langle\epsilon_{n}\right\rangle\right)^{2} \sum_{i}\left(v_{m}^{i}-\left\langle v_{m}\right\rangle\right)^{2}}}
$$

the $\epsilon_{n}^{i}$ and $v_{m}^{i}$ are the values of $\epsilon_{n}$ and $v_{m}$ corresponding to the given event i. $C\left(\epsilon_{n}, v_{n}\right)$ is a decreasing function of the impact parameter for all the harmonics $n$, see Fig.3. We observe that for the second harmonic the correlation coefficient $C\left(\epsilon_{2}, v_{2}\right) \approx 1$ for all the range of impact parameter studied and for both RHIC and LHC. A lower correlation is observed for $n>3$ where for example $C\left(\epsilon_{5}, v_{5}\right)<0.5$ for all centralities. The comparison between dotted an solid lines show that at LHC there is more correlation then at RHIC. In particular at LHC and for ultra-central collisions $C\left(\epsilon_{n}, v_{n}\right)>0.9$ for $n=2,3,4$ as shown by the orange band. This behavior suggest that the value obtained for $\left\langle v_{n}\right\rangle$ are strongly related to the initial value of $\epsilon_{n}$.

\section{Conclusions}

We have studied the effect of the $\eta / s$ on the anisotropic flows $v_{n}\left(p_{T}\right)$ for $n=$ $2,3,4$ and 5 within an event-by-event transport approach. We found that at LHC energies and for mid-peripheral collisions there is a weak sensitivity of $v_{n}\left(p_{T}\right)$ to the $\mathrm{T}$ dependence in the QGP phase. An interesting result we observe for ultra-central collisions where found an enhancement of the sensitivity of the $v_{n}\left(p_{T}\right)$. This sensitivity increase with the order of the harmonics $n$ and for $n=5$ it reaches about a $30 \%$. 
Finally, the study of the correlations between the initial eccentricities $\epsilon_{n}$, and the final anisotropic flows $\left\langle v_{n}\right\rangle$ shows that $C\left(\epsilon_{n}, v_{n}\right)$ is a decreasing function of the impact parameter. Moreover larger is the collision energy larger is the degree of correlation and at LHC there is significantly more correlation than at RHIC. In particular at LHC energies and for ultra-central collisions $(0-0.2 \%)$ we found that $C\left(\epsilon_{n}, v_{n}\right)>0.9$ up to the 4 -th harmonic. This strong linear correlation imply that the $v_{n} \propto \epsilon_{n}$ and it suggests that the study of $v_{n}$ can give information about the initial geometry of the fireball.

\section{Acknowledgments}

V.Greco, S. Plumari, F. Scardina and G.L. Guardo acknowledge the support of the ERC-StG Grant under the QGPDyn project.

\section{References}

[1] Romatschke P and Romatschke U 2007 Phys.Rev.Lett. 99172301.

[2] Song H and Heinz U W 2008 Phys.Rev. C78 024902.

[3] Ferini G, Colonna M, Di Toro M and Greco V 2009 Phys.Lett. B670 325.

[4] Xu Z and Greiner C 2009 Phys.Rev. C79 014904.

[5] Kovtun P, Son D and Starinets A 2005 Phys.Rev.Lett. 94111601.

[6] Qin G Y, Petersen H, Bass S A and Muller B 2010 Phys.Rev. C82 064903.

[7] Holopainen H, Niemi H and Eskola K J 2011 Phys.Rev. C83 034901.

[8] Schenke B, Jeon S and Gale C 2012 Phys.Rev. C85 024901.

[9] Gale C, Jeon S, Schenke B, Tribedy P and Venugopalan R 2013 Phys.Rev.Lett. 110012302.

[10] Csernai L P, Kapusta J and McLerran L D 2006 Phys.Rev.Lett. 97 152303.

[11] Lacey R A, Ajitanand N, Alexander J, Chung P, Holzmann W et al. 2007 Phys.Rev.Lett. 98092301. 
[12] Prakash M, Prakash M, Venugopalan R and Welke G 1993 Phys.Rept. 227321.

[13] Chen J W, Li Y H, Liu Y F and Nakano E 2007 Phys.Rev. D76 114011.

[14] Meyer H B 2007 Phys.Rev. D76 101701.

[15] Das S K and Alam J e 2011 Phys.Rev. D83 114011.

[16] Plumari S, Greco V and Csernai L 2013 arXiv:1304.6566.

[17] Gardim F G, Grassi F, Luzum M and Ollitrault J Y 2012 Phys.Rev. C85 024908.

[18] Chaudhuri A, Haque M R, Roy V and Mohanty B 2013 Phys.Rev. C87 034907.

[19] Niemi H, Denicol G, Holopainen H and Huovinen P 2013 Phys.Rev. C87 054901.

[20] Plumari S, Guardo G L, Scardina F and Greco V 2015 arXiv:1507.05540.

[21] Nakamura A and Sakai S 2005 Phys.Rev.Lett. 94072305.

[22] Schmidt W, Katscher U, Waldhauser B, Maruhn J, Stocker H et al. 1993 Phys.Rev. C47 2782.

[23] Danielewicz P, Barker B and Shi L 2009 AIP Conf.Proc. 1128104.

[24] Arnold P B, Moore G D and Yaffe L G 2003 JHEP 0305051.

[25] Plumari S, Puglisi A, Scardina F and Greco V 2012 Phys.Rev. C86 054902 .

[26] Ruggieri M, Scardina F, Plumari S and Greco V 2013 Phys.Lett. B727 177.

[27] Ruggieri M, Scardina F, Plumari S and Greco V 2014 Phys.Rev. C89 054914 .

[28] Plumari S, Guardo G L, Greco V and Ollitrault J Y 2015 Nucl. Phys. A941 87.

[29] Xu Z and Greiner C 2005 Phys.Rev. C71 064901.

[30] Greco V, Ko C and Levai P 2003 Phys.Rev. C68 034904. 